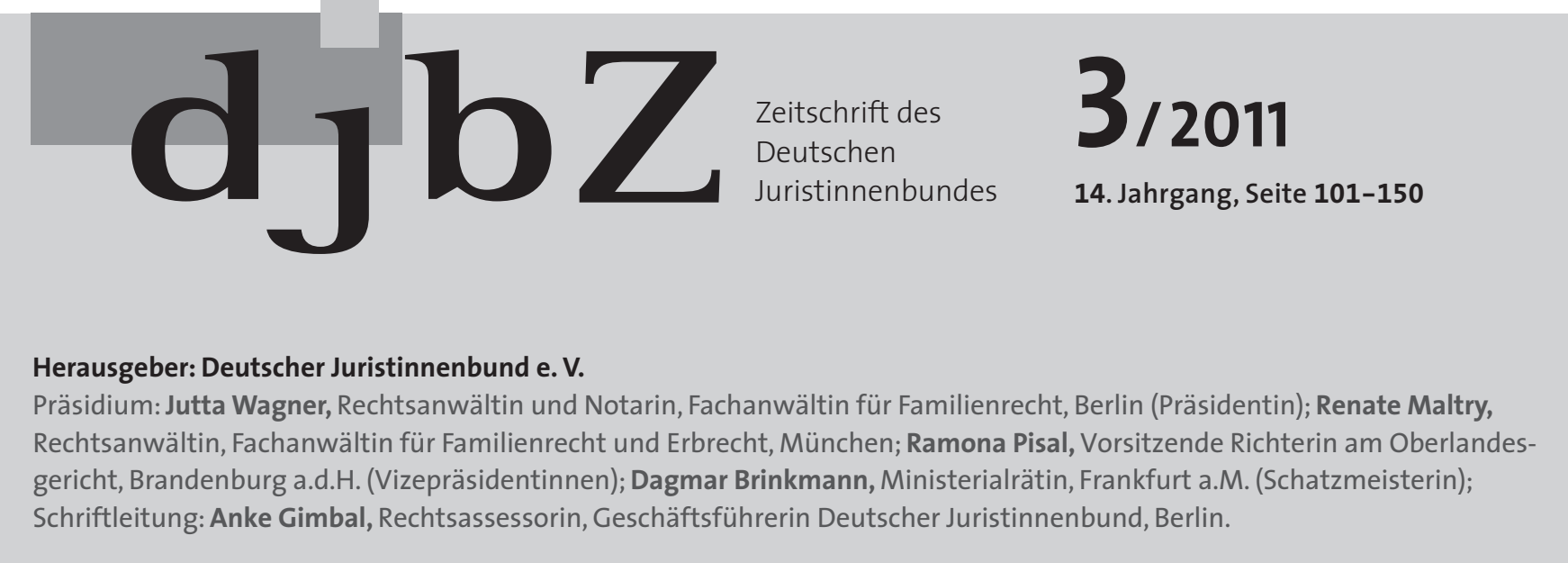

\title{
Das Recht der gemeinsamen Verantwortung für nicht miteinander verheiratete Eltern - Vorschläge zur Gesetzesfassung
}

\section{Dr. Angelika Nake}

Vorsitzende der Kommission Zivil-, Familien- und Erbrecht, Recht anderer Lebensgemeinschaften des djb; Rechtsanwältin, Griesheim

In seiner Entscheidung vom 21. Juli 2010 hat das Bundesverfassungsgericht festgestellt: „Es verletzt das Elternrecht des Vaters eines nichtehelichen Kindes aus Artikel 6 Absatz 2 GG, dass er ohne Zustimmung der Mutter generell von der Sorgetragung für sein Kind ausgeschlossen ist und nicht gerichtlich überprüfen lassen kann, ob es aus Gründen des Kindeswohls angezeigt ist, ihm zusammen mit der Mutter die Sorge für sein Kind einzuräumen oder ihm anstelle der Mutter die Alleinsorge für das Kind zu übertragen. " 1 Eine Frist hat es dem Gesetzgeber ausdrücklich nicht gesetzt. ${ }^{2}$

Bis zum Wirksamwerden eines Gesetzes, welches den Vorgaben des Bundesverfassungsgerichts entspricht, hat das Gericht vorgegeben:

- Daher ist eine - vom bisherigen gesetzlichen Regelungskonzept ausgehende - Übergangsregelung angezeigt.

- Das Familiengericht überträgt den Eltern auf Antrag eines Elternteils die elterliche Sorge oder einen Teil der elterlichen Sorge gemeinsam, soweit zu erwarten ist, dass dies dem Kindeswohl entspricht.

- Das Familiengericht überträgt dem Vater auf Antrag eines Elternteils die elterliche Sorge oder einen Teil der elterlichen Sorge, soweit eine gemeinsame elterliche Sorge nicht in Betracht kommt und zu erwarten ist, dass dies dem Kindeswohl am besten entspricht.

- Es besteht daher für den Gesetzgeber zurzeit keine Not, einen Gesetzentwurf mit Zeitlimit vorzulegen.

\section{Lösungsansätze}

Nun gibt es zwei grundsätzlich unterschiedliche Ansätze in der Frage der Lösung. Zum einen die sogenannte Widerspruchslösung. Hier soll der Vater mit der Vaterschaftsanerkennung automatisch die elterliche Sorge erhalten und die Mutter muss im Fall, dass sie gegenteiliger Ansicht ist, einen Antrag beim zuständigen Familiengericht stellen. Daneben gibt es die sogenannte Antragslösung, die in ihrem Grundkonzept davon ausgeht, dass der Vater einen Antrag bei Gericht stellen muss, wenn er gegen den Willen der Mutter die Mitsorge für das Kind ausüben will.

\section{Widerspruchslösungen}

Als erste Partei ist die FDP mit ihrem Wunsch nach einem „modernen Sorgerecht“ an die Öffentlichkeit gegangen. Ziel war es, die Situation der nichtehelichen Väter zu verbessern. ${ }^{3}$ Die Eltern sollten von Beginn an die gemeinsame Sorge ausüben, wenn die Mutter nicht widerspricht. Bezeichnet wurde dies als einfache Regelung, die den modernen Beziehungsformen angepasst sei. Kinder hätten einen Anspruch darauf, dass Väter Verantwortung übernähmen. Die Justizministerin unterstützte diesen Vorstoß und schlug ebenfalls vor, dass unverheiratete Eltern von Anfang an die gemeinsame Sorge ausüben, es sei denn, die Mutter lege Widerspruch dagegen ein.

Auch die Partei der Linken diskutiert zurzeit den Entwurf des Abgeordneten Jörn Wunderlich, in welchem dieser den Gesetzgeber auffordert, einen Gesetzentwurf vorzulegen, bei

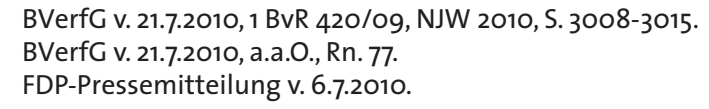


dem die Vaterschaftsanerkennung durch eine gemeinsame Erklärung die gemeinsame Sorge nach sich zieht. Wenn aber eine gemeinsame Erklärung nicht vorliegt, soll die Mitsorge des Vaters aus einem positiven Vaterschaftstest folgen. Ansonsten entsteht die Mitsorge dann, wenn der Vater einen Antrag beim Jugendamt stellt und die Eltern zu diesem Zeitpunkt in einer Lebensgemeinschaft leben.

Argumentativ werden die Widerspruchslösungen immer daran geknüpft, dass es angeblich im europäischen Ausland die „übliche“ Regelung sei und dass eine generelle Schlechterstellung nichtverheirateter Väter gegenüber Müttern und geschiedenen Vätern nicht nur gegen das Diskriminierungsverbot aus Artikel 14 in Verbindung mit Artikel 8 der Europäischen Menschenrechtskonvention (EMRK) verstoßen würde, sondern auch gegen Artikel 6 Absatz 2 des Grundgesetzes. Eine Bevorzugung von geschiedenen Vätern gegenüber getrennt lebenden, mit der Mutter nicht verheirateten Vätern sei indes grundgesetzlich keineswegs geboten.

\section{Antragslösungen}

Die Antragslösungen, die meiner Ansicht nach zurzeit mehrheitlich vertreten werden, gehen immer davon aus, dass die alleinige Sorge zuerst bei der Mutter liegt und erst auf Antrag des nichtehelichen Vaters eine gemeinsame Sorge überhaupt entstehen kann. Eine automatische Mitsorge ohne den Antrag des Vaters, die Mitsorge betreffend, lehnt diese Meinung ab.

Hierzu gehört der Gesetzesvorschlag des djb $b^{4}$ dem Grunde nach und der Gesetzesvorschlag des VAMV. ${ }^{5}$ Auch das Zukunftsforum Familie (ZFF) hat im Mai eine Stellungnahme veröffentlicht, nach der die Antragslösung favorisiert wird. ${ }^{6}$ Das Zukunftsforum hat sich auch ausdrücklich dem Vorschlag der Kommission angeschlossen, die elterliche Sorge in Zukunft in „elterliche Verantwortung “ umzubenennen.

Die Kommission Zivil-, Familien- und Erbrecht, Recht anderer Lebensgemeinschaften des djb hat sich entschlossen eine neue Begrifflichkeit zu fordern, weil insbesondere auch auf europäischer Ebene der Begriff „elterliche Verantwortung “ benutzt wird, der deutlicher macht, dass es bei der elterlichen Sorge auch und insbesondere um Pflichten geht. Der djb hat daher angeregt, in Übereinstimmung mit der Regelung des Artikels 8 der Brüssel-IIa-Verordnung und des Haager Übereinkommens über die Zuständigkeit, das anzuwendende Recht, die Anerkennung, Vollstreckung und Zusammenarbeit auf dem Gebiet der elterlichen Verantwortung und der Maßnahmen zum Schutz von Kindern vom 19. Oktober 1996 (KSÜ), grundsätzlich von elterlicher Verantwortung zu sprechen. ${ }^{7}$

Das ZFF führt in seinem Positionspapier hierzu aus: „Wir treten daher dafür ein, den Begriff des elterlichen „Sorgerechts“ durch jenen der „elterlichen Verantwortung“ zu ersetzen. Für jemanden Verantwortung zu übernehmen und stellvertretend Entscheidungen zu treffen, setzt die Möglichkeit wie die Bereitschaft voraus, sich dieser Verantwortung zu stellen und sie (mit) zu tragen." 8
Dies ist im Grunde auch die Argumentation aller Gegnerinnen und Gegner eines automatischen Sorgerechts, welches alleine aus der Vaterstellung heraus entsteht und nicht an eine gesonderte Willenserklärung des Vaters geknüpft ist, die Verantwortung tragen zu wollen. Der Begriff „Sorgerecht“ kann dazu führen, dass die Vorstellung, man habe ein „Recht am Kind“ dazu verführen kann, dieses vermeintliche Recht einzufordern ohne eine Vorstellung davon zu haben, welche Verantwortung in der Sorge für ein Kind liegt.

\section{Die elterliche Verantwortung für ein Kind}

Immer noch sind 90 Prozent aller alleinerziehenden Elternteile Frauen, ${ }^{9}$ obwohl in den Medien immer wieder die sogenannten „neuen Väter“ beschworen werden. Das Internet bietet Vätern sogar Tests an, mit denen sie feststellen können, ob sie ein guter Vater sind. ${ }^{10}$ In Väterverbänden wird behauptet: „Unser Familienrecht benachteiligt Väter. Deshalb verhalten sich manche Frauen in Familienangelegenheiten vollkommen kompromisslos. Unser Familienrecht verführt Frauen zum Machtmissbrauch. Wer das alleinige Sorgerecht hat, kann seinen eigenen Egoismus hemmungslos ausleben und der staatliche Wächter drückt beide Augen zu. “11 Dementgegen steht eine vom Bundesministerium der Justiz (BMJ) beauftragte Studie zum Sorgerecht nichtverheirateter Eltern. Diese macht deutlich, dass die Erklärung der gemeinsamen Sorge Ausdruck und Ergebnis einer stabilen und tragfähigen Paarbeziehung ist, die Studie warnt vor überhöhten Erwartungen an das gemeinsame Sorgerecht. ${ }^{12}$ Im Gegenteil kommt die Studie sogar eher zu dem Ergebnis, dass sich die Sorgerechtsregelung für die Entwicklung des Kindes als unerheblich erweist. Entscheidend sei hierfür vielmehr das Erziehungsverhalten und die elterliche Kooperation in der Erziehung. ${ }^{13}$

Es ist nicht zu erwarten, dass durch ein gesetzlich vorgesehenes gemeinsames Sorgerecht schwierige und konflikthafte Paarbeziehungen einfacher werden würden oder es gar durch

4 〈http://www.djb.de/Kom/K2/pm10-24GE_Sorgerecht/> (Zugriff: 27.6.2011).

5 Hierzu: <http://www.vamv.de/fileadmin/user_upload/bund/dokumente/Stellungnahmen/Sorgerecht_Formulierungsvorschlag_ des_VAMV_4_11_10.pdf> (Zugriff: 4.7.2011).

6 Positionspapier des ZFF: <http://zukunftsforum-familie.de/ data/ zff_positionspapier_sorgerecht_endg.pdf> (Zugriff: 4.7.2011).

7 <http://www.djb.de/Kom/K2/pm10-24GE_Sorgerecht/> (Zugriff: 4.7.2011).

8 Positionspapier des ZFF, a.a.O.

9 Statistisches Bundesamt, Mikrozensus 2009

10 Z.B. „Lesen Sie auch bei unserem Partner RTL.de: Sind Sie ein guter Vater? Machen Sie den Test! - Testen Sie auf rtl.de Ihre Vaterkompetenzen!", erhältlich im Internet unter <http://www.rtl.de/cms/ ratgeber/familie/sebsttest/sind_sie_ein_guter_vater.html $>$ (Zugriff: 3.7.2011).

11 〈http://www.vaeter-aktuell.de/infomaterial/FAO.htm> (Zugriff: 4.7.2011).

12 Vgl. Jurczyk, Karin/Walper, Sabine, Vorgezogener Endbericht für das Projekt „Gemeinsames Sorgerecht nicht miteinander verheirateter Eltern“, München, 30.11.2010, abrufbar im Internet unter <http:// www.bmj.de/SharedDocs/Kurzmeldungen/DE/2011/20110203_Sorgerecht_Kompromissvorschlag.html?nn=1469052> (Zugriff: 4.7.2011).

13 Vgl. ebd. S. 346. 
eine Sorgeregelung hin zur gemeinsamen Sorge zu einer guten elterlichen Kooperation kommen würde. Genauso wenig ist bei Eltern, die keine gemeinsamen Erziehungsvorstellungen und völlig divergierende Erziehungsansätze haben, zu erwarten, dass sich diese Widersprüche durch die gesetzliche Anordnung einer gemeinsamen Sorge auflösen werden.

Es muss von Anfang an klar sein, dass es sich bei der Sorge um die gemeinsame elterliche Verantwortung handelt und dies eine „Bringschuld für den Vater“ darstellt. Wesentlich ist in diesem Zusammenhang insbesondere die kindeswohldienliche Kooperation zwischen den Eltern, die Fähigkeit der Eltern, über die Bedürfnisse und Belange des Kindes sachlich kommunizieren zu können, selbstverständlich auch eine kooperative Wahrnehmung des Umgangsrechts in kindeswohlgerechter Weise. Nur so kann überhaupt eine tragfähige Beziehung zum Kind aufgebaut werden und nur mit einer tragfähigen Bindung und der Kenntnis von den Bedürfnissen und Fähigkeiten des Kindes kann ein Vater, der nichtehelich ist und womöglich mit dem Kind nie zusammengelebt hat, überhaupt Entscheidungen treffen, die sich am Charakter und den Fähigkeiten des Kindes orientieren können. Bei Eltern, die nie eine Paarbeziehung miteinander eingegangen sind und in Beziehungen, in welchen der Vater niemals mit der Mutter und/oder mit dem Kind zusammengelebt hat, ist es schwieriger für den Vater, Entscheidungen für das Kind zu treffen.

\section{Zusammenleben der nichtehelichen Eltern}

Diese Einsicht führt dann auch dazu, dass die weiteren politischen Ansätze zur Lösung dieser Fragen an das Zusammenleben der Eltern anknüpfen. So knüpft der Vorschlag der Bundestagsabgeordneten der SPD Dagmar Ziegler und Caren Marks sowie auch der Vorschlag der Bundestagsabgeordneten Katja Dörner, Ingrid Hönlinger und Monika Lazar (Bündnis 90/Die Grünen) dann auch an ein gemeinsames Zusammenleben der nichtehelichen Eltern an. Maßstab soll das SGB II sein bzw. das Zusammenleben von zehn Monaten. Charme dieses Lösungsansatzes ist es, dass sich - wie auch die Studie des BMJ festgestellt hat - bei den mitsorgeberechtigten nichtehelichen Eltern, die in einer nichtehelichen familiären Beziehung leben, eine egalitärere Aufgabenverteilung und teilweise das Konzept einer gemeinsamen Verantwortung für das Kind häufig findet. ${ }^{14}$ Die Studie führt aber hierzu weiter aus: „In Bezug auf die Wirkungsrichtung verweisen die qualitativen Interviews allerdings darauf, dass die bessere Kooperation der Eltern eher ein Grund für die Abgabe der Sorgeerklärung als ein Resultat derselben ist. Insofern sollte das gemeinsame Sorgerecht nicht mit überhöhten Erwartungen befrachtet werden."15 Allein daraus, dass die Eltern zusammenleben, kann somit nicht geschlossen werden, dass die Eltern auch kommunikativ und kooperativ im Rahmen der Sorge miteinander umgehen können. Das Zusammenleben ist kein Garant für eine kindeswohlorientierte Ausübung der elterlichen Verantwortung. Das Ergebnis der Studie ist dann auch: „Zusammengenommen lassen diese
Befunde schlussfolgern, dass ein gemeinsames Sorgerecht, das für zusammenlebende Paare ab Geburt des Kindes - ggf. je nach Dauer des Zusammenlebens - eingerichtet wird, angemessen erscheint. (...) Eine generelle Zuweisung des gemeinsamen Sorgerechts auch für getrennt lebende Elternpaare oder Eltern, die keine gemeinsame Partnerschaft haben, erscheint demgegenüber weniger empfehlenswert. “16

Die Studie knüpft eher an eine Beziehung zwischen den Eltern als an ein Zusammenleben an. Hierbei stellt sich dann aber die Frage, ob hierfür ein gemeinsamer Wohnsitz im Sinne des SGB II aussagekräftig ist. Nachdem die Meldegesetze heute nicht mehr den Nachweis des tatsächlichen Wohnsitzes voraussetzen, wie Bescheinigungen der Vermieter, führt alleine der Wohnsitz nicht mehr zu dem Beweis, dass der dort Gemeldete auch dort tatsächlich wohnt. Der Beweis, dass die beiden Eltern auch eine Beziehung führen, kann hierdurch erst recht nicht geführt werden. Die Anknüpfung an einen Automatismus, wenn ein gemeinsamer Wohnsitz vorliegt, könnte dazu führen, dass die Mutter von der Rechtsfolge der gemeinsamen Sorge gar keine Kenntnis haben könnte. Auch der nichteheliche Vater, der eine reine Wohngemeinschaft mit der Mutter hat, geht sicherlich nicht davon aus, dass er die Mitsorge hat.

Darüber hinaus ist für das gemeinsame Sorgerecht der ehelichen Kinder das Zusammenleben der Eltern nicht Voraussetzung. Eheliche Eltern haben das gemeinsame Sorgerecht auch in dem Fall, dass sie in verschiedenen Staaten wohnen. Es steht daher hier in Frage, ob dies nicht eine ebenfalls grundrechtswidrige Lösung ist.

\section{Widerspruchslösung des Bundesjustizministeriums}

Dem Kompromissvorschlag des BMJ vom 3. Februar $2011^{17}$ zufolge hätte die Mutter bei der Geburt des Kindes zunächst das alleinige Sorgerecht. Erklärt der nicht mit der Mutter verheiratete Vater durch Abgabe einer Sorgeerklärung allerdings, dass er mit der Mutter gemeinsam die elterliche Sorge ausüben will, hat die Mutter acht Wochen Zeit, sich eine Meinung über die gemeinsame Sorge zu bilden. Äußert sich die Mutter nicht, entsteht nach acht Wochen kraft Gesetzes die gemeinsame Sorge. Widerspricht die Mutter der gemeinsamen Sorge, hat der Vater die Möglichkeit, einen Antrag beim Familiengericht zu stellen. Das Gericht hätte dann zu entscheiden, ob das gemeinsame Sorgerecht dem Kindeswohl widerspricht oder nicht.

Das Ministerium knüpft die Rechtsfolge des gemeinsamen Sorgerechts an ein Schweigen der Mutter: „Wenn jedoch die Vaterschaft geklärt ist und der Vater durch eine Sorgeerklärung verdeutlicht, dass er Verantwortung für das Kind übernehmen will, erscheint auf der zweiten Stufe die

\footnotetext{
14 S. 352 des Berichts, a.a.O.

15 Ebd.

16 S. 352-353, a.a.O.

17 Im Internet erhältlich: <http://www.bmj.de/SharedDocs/Kurzmeldungen/DE/2011/20110203_Sorgerecht_Kompromissvorschlag. html?nn=1469052> (Zugriff: 4.7.2011).
} 
Entstehung der gemeinsamen Sorge sinnvoll, wenn die Mutter innerhalb von acht Wochen keine Bedenken äußert."18

Fraglich ist allerdings schon der Ansatzpunkt, dass es sich hierbei um ein Antragsmodell handelt. Das Antragsmodell setzt zwar zwingend einen Antrag des Vaters voraus, die Sorge für sein Kind ausüben zu wollen, das Antragsmodell geht aber dann davon aus, dass die Mutter sich hierzu positiv oder negativ äußert. Der Vorschlag des BMJ geht aber hiervon nicht aus. Auch in dem Fall, in dem sich die Mutter nicht äußert, entsteht ein gemeinsames Sorgerecht - quasi automatisch. Das Modell des BMJ geht zwar davon aus, dass die Mutter von dem Antrag des Vaters Kenntnis hat und sich innerhalb der gesetzten Frist von acht Wochen nicht äußert. Mit keinem Wort äußert es sich aber dazu, wie es die Zustellung des Antrags an die Mutter sicherstellen will.

Wenn das Jugendamt zustellt, befinden wir uns im verwaltungsrechtlichen Bereich. Voraussetzung dafür, dass hier eine Frist überhaupt zu laufen beginnt, ist nicht nur die wirksame Zustellung (Zustellungsurkunde oder Einschreiben/ Rückschein oder Einwurfeinschreiben oder Fax), sondern auch und insbesondere eine wirksame Rechtsbehelfsbelehrung. Wenn diese nicht vorliegt, beginnt die Frist nicht zu laufen bzw. es ist auch noch bis zu einem Jahr möglich, die Rechtshandlung nachzuholen. Im Übrigen kann im Verwaltungsrecht der Verwaltungsakt sogar drei Tage nach Aufgabe zur Post als wirksam zugestellt gelten. Was gilt bei einer $\mathrm{Zu}-$ stellung ins benachbarte europäische Ausland und wer trägt hier die Folgen einer nicht wirksamen Zustellung? So wie der Vorschlag des BMJ zu lesen ist, erhält der Vater das Sorgerecht nach Ablauf der acht Wochen Wartefrist automatisch. Das kann aber dazu führen, dass die Mutter womöglich keine Kenntnis von dem Antrag hat und von der Mitsorge des nichtehelichen Vaters erst erfährt, wenn dieser die ersten Entscheidungen getroffen hat oder sein Veto gegen Entscheidungen einlegt, die die Mutter trifft.

Eine Rechtsfolge, die so tief greifende Rechtswirkungen für die Beteiligten hat, kann nicht an ein Schweigen geknüpft werden. Zumal die deutsche Rechtsordnung den positiven Erfolg einer Willenserklärung durch Schweigen außer im Fall der Kaufmannseigenschaft nicht kennt. Die Rechtsfolge wäre noch ungewisser als im Falle der reinen Widerspruchslösung.

Auch der Gesetzesvorschlag der Grünen ${ }^{19}$ knüpft daran an, dass der Vater die Vaterschaft anerkennt oder die Vaterschaft gerichtlich festgestellt wird und das Jugendamt unter Fristsetzung von acht Wochen die Mutter zur Stellungnahme auffordert. Auch hier wird das Sorgerecht erteilt, wenn die Mutter innerhalb der Frist nicht widerspricht und keine Kindeswohlgefährdung zu erkennen ist. Auch diese Lösung ist aus Gründen des Rechtschutzes für das Kind daher abzulehnen. Unabhängig dazu wird darauf hingewiesen, dass auch die Hemmung der Frist durch Mutterschutzfristen keine Rechtssicherheit gewährt, weil die Länge der sogenannten Mutterschutzfrist divergiert, je nachdem, ob es sich um eine
Mehrlingsgeburt oder um eine Frühgeburt handelt. Auch hier ist die Rechtsfolge nicht sicher und für die Beteiligten nicht ausreichend sicher vorherzusehen.

\section{Vorschlag des djb}

Nach dem djb-Vorschlag - der differenzierten Widerspruchslösung - ist die Rechtsfolge der gemeinsamen elterlichen Verantwortung wie folgt ausgestaltet:

\1626a BGB, Differenzierte Widerspruchslösung

(1) Sind die Eltern bei der Geburt des Kindes nicht miteinander verheiratet, so steht ibnen die elterliche Sorge dann gemeinsam zu, wenn

a. der Vater erklärt, dass er die elterliche Verantwortung übernehmen will und

b. die Mutter erklärt, dem nicht zu widersprechen.

(2) Liegen die Voraussetzungen des Absatzes 1 nicht vor, so hat das Familiengericht auf Antrag eines Elternteils die gemeinsame Sorge anzuordnen, wenn es dem Kindeswohl nicht widerspricht.

(3) Im Übrigen hat die Mutter die elterliche Sorge.

Die Übernahme der elterlichen Verantwortung für ein Kind setzt nach Ansicht der djb-Kommission zwingend voraus, dass es wenigstens ein Minimum an Kooperationswillen und Kooperationsbereitschaft gibt, nämlich die Bereitschaft, gemeinsam Verantwortung für das Kind zu tragen. Die Ausübung dieser gemeinsamen Verantwortung erfordert den Aufbau einer persönlichen Beziehung zum Kind durch jeden Elternteil und bedarf eines Mindestmaßes an Übereinstimmung zwischen den Eltern. Fehlt es hieran und sind die Eltern zur Kooperation weder bereit noch in der Lage, kann die gemeinsame Sorge für das Kind dem Kindeswohl zuwiderlaufen. ${ }^{20}$

Auch die Argumentation mit anderen europäischen Staaten ${ }^{21}$ kann zu einer Lösungsfindung nur begrenzt beitragen, da das Rechtswesen und die Rechtswirklichkeit bei einem einfachen Vergleich nicht in Betracht gezogen werden kann. So liegt beispielsweise das Sorgerecht in Spanien und Portu-

18 Ebd.

19 BT-Drs. $17 / 3219$ v. 6.10.2010, erhältlich im Internet: 〈http://dip21. bundestag.de/dip21/btd/17/032/1703219.pdf (Zugriff: 4.7.2011). 20 So bereits BVerfGE 107, $150\langle 173\rangle$.

21 Finnland: $\$ \S 9,10$ finn. SorgRG; Irland: Sec. 6A para 1 Guardianship of Infants Act 1964 i.d.F. der Sec. 6 Children Act 1997, Sec. 9 Guardianship of Infants Act 1964; Luxemburg: Art. 380 lux. CC; Niederlande: Art. 1:253C nl. BW; Schweden: 6:5 schwed. FB; Vereinigtes Königreich: Sec. 4 para. 1c Children Act 1989; Zypern: Sec. 6 Illegitimate Children Law CAP 278). In 18 EU-Mitgliedstaaten sind unverheiratete Eltern verheirateten Eltern weitgehend oder vollständig gleichgestellt und erlangen kraft Gesetzes das gemeinsame Sorgerecht (Belgien: Art. 373, Art. 374 § 1 belg. CC; Bulgarien: Art. 68 Abs. 1, Art. 72 bulg. FamGB; Dänemark: Kap. $2 \oint 7$ des dän. Gesetzes über elterliche Verantwortung; Estland: $\$ \S 49,50$ estn. FamG; Frankreich: Art. 372 franz. Cciv; Griechenland: Art. 1515 griech. ZGB; Italien: Art. 317bis ital. Cciv; Lettland: Art. 178, 181 lett. ZGB; Litauen: Art. 3.165 litau. ZGB; Malta: Art. 90 Abs. 1, Art. 86 malt. ZGB; Polen: Art. $93 \S 1$ poln. FVGB; Portugal: Art. 1911 i.V.m. Art. 1901 port. CC; Rumänien: Art. 97 rumän. FGB; Slowakei: § 28 Abs. 2 slowak. FamG; Slowenien: Art. 102, Art. 105 Abs. 1 slowen. EheFamG; Spanien: Art. 156 span. CC; Tschechische Republik: § 34 Abs. 1, §52 Abs. 1 tschech. FamG; Ungarn: $\$ 72$ Abs. 1 ungar. FamG). 
gal bei dem Elternteil, bei dem das Kind lebt, unabhängig ob dies Mutter oder Vater ist. In Belgien gibt es eine Art Vermutung dafür, dass Entscheidungen, die durch den Elternteil getroffen werden, bei dem das Kind lebt, wirksam sind und diese Entscheidungen auch gegenüber dem anderen Elternteil gelten. Diese Einzelaspekte werden bei den rechtsvergleichenden Betrachtungen aber regelmäßig nicht in Erwägung gezogen.

So führte dann in Deutschland eine Mitsorge dazu, dass der nichteheliche Vater - gemeinsam mit der Richterin oder dem Richter - entscheiden kann, dass das Kind eine bestimmte Schule oder einen bestimmten Kindergarten besucht oder gar eine Religionszugehörigkeit erhält, die die nichteheliche Mutter- bei der das Kind lebt - dann ausführen muss. Dies würde dazu führen, dass der nichteheliche Vater in das Leben der nichtehelichen Mutter in einer Weise hineinregiert, die deren privateste Lebensbereiche betrifft.

Bei solchen Auswirkungen kann ein Automatismus nicht wünschenswert sein. Verfassungsrechtlich geboten ist dieser keinesfalls. ${ }^{22}$ Das Bundesverfassungsgericht weist ausdrücklich darauf hin, dass der Gesetzgeber in seine Erwägungen einbeziehen darf, dass eine generelle gesetzliche Anordnung der gemeinsamen elterlichen Sorgetragung auch Fälle umfassen kann, in denen aufgrund massiver Konflikte zwischen den Eltern das Kindeswohl zumindest so lange in Mitleidenschaft gezogen würde, bis die gemeinsame Sorge der Eltern durch gerichtliche Entscheidung wieder aufgehoben und in eine Alleinsorge überführt würde. ${ }^{23}$ Um dies zu verhindern, ist es in Abwägung des Kindeswohls mit dem Elternrecht beider Elternteile ebenfalls verfassungsrechtlich gerechtfertigt und nicht zu beanstanden, dass der Gesetzgeber davon abgesehen hat, dem Vater eines nichtehelichen Kindes mit wirksamer Vaterschaftsanerkennung zugleich kraft Gesetzes die gemeinsame Sorge mit der Mutter zu übertragen, womit es auch bei erfolgter Anerkennung der Vaterschaft zunächst bei der alleinigen Sorgetragung für das Kind durch die Mutter verbleibt. $^{24}$

Auch ein automatisches Sorgerecht führt nicht dazu, dass Gleichberechtigung dergestalt eintreten würde, dass in allen Fällen direkt mit der Geburt sowohl Vater wie auch Mutter die gemeinsame elterliche Verantwortung haben, da in vielen Fällen die Vatereigenschaft des nichtehelichen Vaters erst geklärt werden muss. In diesen Fällen muss die Mutter die alleinige Sorge haben, da das Kind eine Person benötigt, die wichtige Entscheidungen für es treffen kann. Ein Sorgeschwebezustand wäre hier kindeswohlfeindlich.

Auch kann einem nichtehelichen Vater nicht gegen seinen Willen die elterliche Verantwortung aufgezwungen werden, da die Nichttätigkeit des Vaters und die Verweigerung von Entscheidungen, die elterliche Verantwortung betreffend, ebenfalls kindeswohlfeindlich ist. ${ }^{25}$ Aus diesem Grunde ist es auch für die Feststellung der Mitsorge zwingend, dass der Vater positiv bestätigt, die elterliche Verantwortung zusammen mit der Mutter tragen zu wollen. Der nichtehelichen Mutter wird hier nur abverlangt, dass sie ausdrücklich be- stätigt, nicht zu widersprechen. Angesichts des Zeitpunktes, zu welchem eine ausdrückliche Stellungnahme von der Mutter abverlangt wird, nämlich relativ zeitig nach der Geburt, würde eine Zustimmung die Anforderungen in den Fällen verschärfen, in denen es vorher keine Beziehung zwischen den Eltern gegeben hat, die Mutter daher den Vater wenig kennt, aber auch keine Einwände gegen ihn als Vater hat.

Die Schwelle der Widerspruchslösung des djb ist daher niedriger als die Schwelle einer ausdrücklichen Zustimmung zur gemeinsamen elterlichen Verantwortung. Eine Fristenlösung mit den oben genannten Nachteilen und Schwierigkeiten wird hierdurch vermieden. Wenn die Mutter die Zustimmung verweigert bzw. Widerspruch erhebt, kann der nichteheliche Vater Klage einreichen. Die Mutter muss dann aber begründen, warum die Mitsorge des nichtehelichen Vaters dem Kindeswohl widerspricht. Wichtig ist der Kommission auch, dass beide Eltern bei Gericht auf Mitsorge klagen können, auch die Mutter kann Interesse daran haben, dass der nichteheliche Vater die elterliche Verantwortung mitträgt.

Die differenzierte Widerspruchslösung des djb ist daher eine interessengerechte und am Kindeswohl orientierte vermittelnde Lösung. 